\title{
Dopaminergic Correlates of Sensory-Specific Satiety in the Medial Prefrontal Cortex and Nucleus Accumbens of the Rat
}

\author{
Soyon Ahn and Anthony G. Phillips \\ Department of Psychology, University of British Columbia, Vancouver, British Columbia, Canada V6T $1 Z 4$
}

Changes in dopamine (DA) efflux in the medial prefrontal cortex and nucleus accumbens of rats were monitored using in vivo microdialysis during sensory-specific satiety experiments. Rats consumed significant amounts of a palatable food during an initial meal but ate little when the same food was available as a second meal. In contrast, rats given a different palatable food ate a significant quantity during the second meal. DA efflux in both brain regions reflected this difference in food intake, indicating that DA activity is influenced by changes in the depriva- tion state of animals and sensory incentive properties of food. Given the proposed role of DA in motivated behaviors, these findings suggest that DA efflux may signal the relative incentive salience of foods and thus is a determinant of the pattern of food consumption observed in sensory-specific satiety.

Key words: sensory-specific satiety; dopamine; medial prefrontal cortex; nucleus accumbens; in vivo microdialysis; rat; appetitive and consummatory phases of feeding behavior; incentive motivation
Sensory-specific satiety is a critical factor in the selection of a varied diet by animals (Berridge, 1991; Rolls, 1999) and humans (Johnson and Vickers, 1992; Hetherington and Rolls, 1996; Rolls and Rolls, 1997). When hungry animals are allowed to satiate on one palatable food, they continue to display satiety when given the same food again, whereas those given a novel food with different sensory properties consume a second meal (Rolls et al., 1986; Berridge, 1991).

A neural mechanism underlying sensory-specific satiety for taste has been proposed based on extensive data from electrophysiological recordings of neurons in the taste pathway as well as key cortical and subcortical regions involved in motivated behaviors. Rolls (1999) argues that neurons critical for the computation of sensory-specific satiety in the monkey are located in the primate orbitofrontal cortex. These neurons are not only finely tuned in their response to specific tastes, they are also affected by internal satiety signals, including gastric distention and glucose utilization. As a result of such modulation, these neurons decrease their firing rates in response to tasting a specific palatable food that has been eaten to satiety but increase their firing rates when the monkey consumes a different food (Rolls et al., 1986). This phenomenon cannot be accounted for by adaptation of taste receptors or indeed of any neuron in the taste pathway, from the nucleus of the tractus solitarius to the primary taste cortex. Rolls (1999) concludes that only after many stages of information processing does the taste system interface to motivation systems in the orbitofrontal cortex. Furthermore, Rolls (1999) suggests that by communicating with the orbitofrontal cortex, regions involved in behavioral output such as the ventral striatum can use

\footnotetext{
Received May 14, 1999; revised July 15, 1999; accepted Aug. 2, 1999.

This research was supported by Group Grant PG12808 from the Medical Research Council (MRC) of Canada and an MRC Canada studentship to S.A. We gratefully acknowledge the contribution of Dr. A. Coury in supervising the HPLC assays.

Correspondence should be addressed to Anthony G. Phillips, Department of Psychology, University of British Columbia, 2136 West Mall, Vancouver, British Columbia, Canada V6T 1 Z4.

Copyright $(\subset) 1999$ Society for Neuroscience $\quad 0270-6474 / 99 / ? ? 0001-\bullet \$ 05.00 / 0$
}

the highly specific reward-related information to guide appropriate behavioral responses.

An equally important, but as yet undetermined, aspect of the sensory-specific satiety mechanism may involve the mesotelencephalic dopamine (DA) system. Its role as a neural substrate of motivation is widely recognized (Blackburn et al., 1992; Kiyatkin, 1995; Robbins and Everitt, 1996; Salamone et al., 1997; Beninger and Miller, 1998; Di Chiara, 1998). In particular, DA activity is believed to mediate appetitive or approach behaviors triggered by incentive stimuli associated with rewards (Fibiger and Phillips, 1986; Berridge and Robinson, 1998). Berridge and Robinson (1998) maintain that phasic increases in DA activity endow immediate salience to biologically important stimuli in the environment, thus prompting animals to select and engage in appropriate patterns of behavioral responses. Consistent with this view, in vivo monitoring studies in rats have demonstrated that increases in DA efflux accompany both appetitive and consummatory phases of feeding in terminal regions of the DA system, such as the nucleus accumbens (NAC) (Phillips et al., 1993; Wilson et al., 1995) and medial prefrontal cortex (mPFC) (Cenci et al., 1992; Feenstra and Botterblom, 1996; Taber and Fibiger, 1997).

A recent report by Bassareo and Di Chiara (1997) emphasizes important differences in DA efflux in the NAC and mPFC in response to food reward and associated incentive stimuli. Specifically, rats fed ad libitum on rat chow display a significant increase in DA efflux in both the NAC and $\mathrm{mPFC}$ when eating a novel

This article is published in The Journal of Neuroscience, Rapid Communications Section, which publishes brief, peerreviewed papers online, not in print. Rapid Communications are posted online approximately one month earlier than they would appear if printed. They are listed in the Table of Contents of the next open issue of JNeurosci. Cite this article as: JNeurosci, 1999, 19:RC29(1-6). The publication date is the date of posting online at www.jneurosci.org.

http://www.jneurosci.org/cgi/content/full/3509 
palatable food. This effect is greatly attenuated in the NAC, when rats ingest the novel food 2-48 hr earlier. Furthermore, DA efflux in the shell of the NAC of nondeprived rats does not increase in response to either a visual or olfactory incentive stimulus associated with palatable food. In contrast, both sensory modalities of incentive stimuli evoke a significant increase in DA efflux in the mPFC. On the basis of these data, Di Chiara (1998) proposes that mesolimbic DA transmission does not code for general motivational salience as hypothesized by Berridge and Robinson (1998) but instead reflects an associative learning process through which novel or motivationally relevant stimuli (under food deprivation conditions) gain the ability to elicit appetitive responses. Motivational salience is a property attributed to the mesocortical DA system.

The present study sought to address three main issues by monitoring changes in dialysate DA levels from the rat $\mathrm{mPFC}$ and NAC during a sensory-specific satiety protocol. First, given that the mPFC (Fuster, 1991; Goldman-Rakic, 1995) and NAC (Mogenson et al., 1993) are key sites for integration of information influencing behavioral response systems and that they are major sites of dopaminergic innervation, we tested the possibility that sensory information may interface to motivational systems at these sites; that is, DA efflux in these regions was predicted to reflect changes in the incentive value of food as modulated by deprivation state of the animal and sensory incentive properties of food. Second, specific features of this protocol may address the question of whether previous opportunity to learn about the incentive value of food reward (i.e., incentive learning; Balleine and Dickinson, 1998) is a critical determinant of increased DA efflux in response to food-related incentive stimuli. Third, we hoped to provide further information on the differences and similarities of DA function in the mPFC and NAC during appetitive and consummatory phases of feeding.

\section{MATERIALS AND METHODS}

Subjects and surgery. Long-Evans male rats (280-310 gm; Charles River Canada, St. Constant, Quebec, Canada) were each implanted bilaterally with stainless steel guide cannulae (10 gauge, $15 \mathrm{~mm}$ ) under xylazine ( 7 $\mathrm{mg} / \mathrm{kg}$, i.p.) and ketamine hydrochloride $(100 \mathrm{mg} / \mathrm{kg}$, i.p.) anesthesia. Cannula tips were located $1 \mathrm{~mm}$ below dura, directly over the NAC $[+1.7$ $\mathrm{mm}$ anteroposterior (AP) and $\pm 1.1 \mathrm{~mm}$ mediolateral (ML) from bregma, $-8.0 \mathrm{~mm}$ dorsoventral (DV) from dura] in 14 animals and the mPFC $(+3.0 \mathrm{~mm} \mathrm{AP}$ and $\pm 0.6 \mathrm{~mm}$ ML from bregma, $-4.5 \mathrm{~mm} \mathrm{DV}$ from dura) in another 14 animals. All animals with mPFC or NAC implants underwent identical training and testing procedures. These experiments were conducted in accordance with guidelines of the Canadian Council of Animal Care and were approved by the Committee on Animal Care, University of British Columbia.

Animals were housed individually in a $21^{\circ} \mathrm{C}$ reverse light cycle colony room (lights off 7 A.M.-7 P.M.). One week after surgery, animals were placed on a restricted feeding schedule, which maintained their body weight at $\sim 85 \%$ free feeding weight, for the duration of the experiment. Each day, animals were weighed and then fed (20 gm Rat Diet 5012; PMI Feeds, Delta, British Columbia, Canada) in their home cages. Water was available at all times except during behavioral test sessions. Novel objects (e.g., sterilized egg and milk cartons) were introduced into the animals' home cages weekly to promote exploratory and play behavior.

Behavioral apparatus and paradigm. Experiments took place a Plexiglas chamber divided by a removable perforated Plexiglas screen. At the beginning of a session, animals were placed into one compartment of the chamber $(26 \times 38 \times 38 \mathrm{~cm})$. Removing the screen allowed access into the adjacent compartment $(16 \times 38 \times 38 \mathrm{~cm})$ where the food bin was located.

The foods used in the sensory-specific satiety experiments were Froot Loops (Kellogg Canada Inc., London, Ontario, Canada) and Onion Rings (Nong Shim Co. Ltd., Seoul, Korea). Both foods gave off strong and distinctive odor cues that were readily detectable at a distance of 1-2 $\mathrm{m}$ by human observers. The foods were similar in caloric content (4 $\mathrm{cal} / \mathrm{gm}$ of Froot Loops and $5 \mathrm{cal} / \mathrm{gm}$ of Onion Rings) and, although very different in sensory properties (e.g., odor and taste), were equally palatable to the animals (pilot data showed that approximately equal amounts were consumed within a meal). The two foods were designated foods 1 and 2, with the designations being counterbalanced within each of the mPFC- and NAC-implanted groups.

Two weeks into the restricted feeding schedule, animals were placed in the testing chamber and given $20 \mathrm{~min}$ to eat food 1 on day 1 and food 2 on day 2 . On day 3 , rats were exposed to a trial run of a sensory-specific satiety procedure that consisted of two meals separated by a $40 \mathrm{~min}$ recess during which no food was available. At the start of the first meal, $3 \mathrm{gm}$ of food 1 was presented in the food bin behind a perforated screen for $10 \mathrm{~min}$ (the appetitive period). The screen was then removed, and animals had access to the food for $10 \mathrm{~min}$, after which the remaining food, if any, was replaced by another $3 \mathrm{gm}$ of the same food every $10 \mathrm{~min}$ for a total of $40 \mathrm{~min}$ (the consummatory period). The recess was followed by presentation of a second meal, in the same manner, of food 1 for half of the animals and food 2 for the other half of the animals.

Microdialysis experiments. Experiments were conducted on days 5 and 7 using the sensory-specific satiety procedure followed on day 3. Each animal served as a subject on both experiment days; one session involved presentation of food 1 for both meals (same food condition), and the other session involved presentation of food 1 for the first meal and food 2 for the second meal (different food condition). The order of testing under the two conditions was counterbalanced. For each animal, one hemisphere was used for the experiment on day 5 , and the other hemisphere was used for the experiment on day 7. The use of right and left hemispheres was randomized across the test conditions.

Fourteen to $16 \mathrm{hr}$ before the start of each experiment, concentric microdialysis probes $(2 \mathrm{~mm}$ membrane length, $340 \mu \mathrm{m}$ outer diameter, 65,000 molecular weight cutoff; Filtral 12; Hospal, Nuernberg, Germany) with silica inlet-outlet lines were implanted unilaterally either in the $\mathrm{mPFC}$ or NAC. Probes were perfused at $1 \mu \mathrm{l} / \mathrm{min}$ with a modified Ringer's solution (10 mM sodium phosphate, $1.2 \mathrm{mM} \mathrm{CaCl}_{2}, 3.0 \mathrm{~mm} \mathrm{KCl}$, $1.0 \mathrm{~mm} \mathrm{MgCl} 2,147.0 \mathrm{~mm} \mathrm{NaCl}, \mathrm{pH} 7.4)$ using a $2.5 \mathrm{ml}$ gas-tight syringe (Hamilton, Reno, NV) and a syringe pump (model 22; Harvard Apparatus, South Natick, MA). Perfusion was continuous from before implantation until the end of the experiment. Implanted animals, fed earlier in the day, remained overnight in the test chamber. Dialysis samples were collected at $10 \mathrm{~min}$ intervals from the mPFC or NAC and analyzed immediately. Baseline conditions continued until four consecutive samples showed $<5 \%$ fluctuation in DA content before the first meal was presented. The equal rate of sampling $(1 \mu \mathrm{l} / \mathrm{min}$ for $10 \mathrm{~min})$ in both brain regions allowed dialysate DA levels from each region to be compared sample-to-sample across time.

HPLC. HPLC with electrochemical detection was used in the analysis of dialysate DA levels from the mPFC. The HPLC system consisted of a Millipore (Milford, MA) Waters 515 HPLC pump, a Scientific Systems Inc. (State College, PA) LP-21 pulse damper, an LC Packings (San Francisco, CA) Acurate splitter and Hypersil $\mathrm{C}_{18}$ BDS column $(5 \mathrm{~cm} \times$ $0.8 \mathrm{~mm}$ inner diameter, $3 \mu \mathrm{m}$ particles), a Valco (Houston, TX) Cheminert $\mathrm{C} 1$ microbore injector ( $5 \mu \mathrm{l}$ loop), a Millipore Waters 460 electrochemical detector modified with an Antec (Leyden, The Netherlands) VT-03 electrochemical flowcell and Hy-REF electrode $\left(\mathrm{V}_{\text {applied }}=+400\right.$ $\mathrm{mV}$ ), and Millipore Maxima analysis software. The mobile phase [ $43 \mathrm{~mm}$ citric acid, $110 \mathrm{~mm}$ sodium acetate, $550 \mathrm{mg} / 1$ sodium octyl sulfate, 100 $\mathrm{mg} / \mathrm{l}$ sodium EDTA dihydrate, 5\% (v/v) methanol, $\mathrm{pH}$ 3.5] flowed through the system at $0.25 \mathrm{ml} / \mathrm{min}$. The HPLC system used to analyze samples from the NAC has been described previously (Fiorino et al., 1997).

Histology. Animals were anesthetized with chloral hydrate and perfused intracardiacally with $0.9 \% \mathrm{NaCl}$ and then $3.7 \%$ paraformaldehyde. Brains were removed and stored in formalin with $20 \%$ (w/v) sucrose for a few days, sliced into $50 \mu \mathrm{m}$ coronal sections, and then stained with cresyl violet. Only data obtained from those animals with tracts in the prelimbic-infralimbic region of the $\mathrm{mPFC}$ or in the shell-core region of the NAC of both hemispheres were included in the statistical analyses.

Data analyses. Neurochemical data were transformed into percent change from $100 \%$ baseline, where $100 \%$ represented the average concentration of the three samples preceding the final baseline sample. Data were analyzed using an ANOVA followed, when appropriate, by the Dunnett or Tukey method of multiple comparisons. The Huynh-Feldt correction for nonsphericity was applied to the degrees of freedom for all within-subject analyses. 


\section{Medial Prefrontal Cortex}

A. Same Food Condition

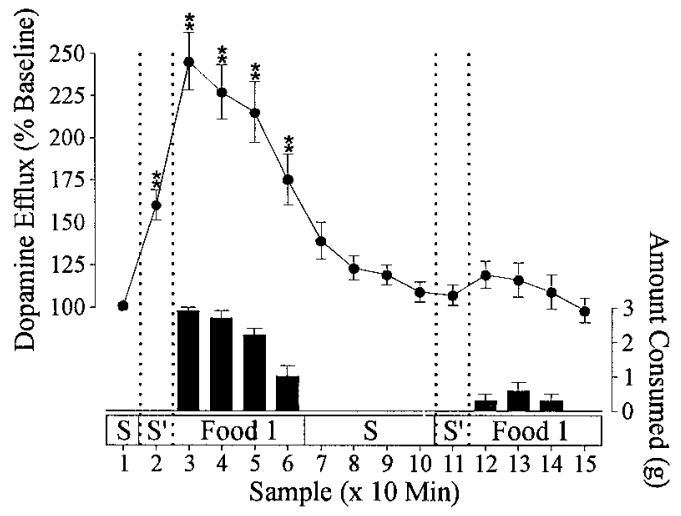

B. Different Food Condition

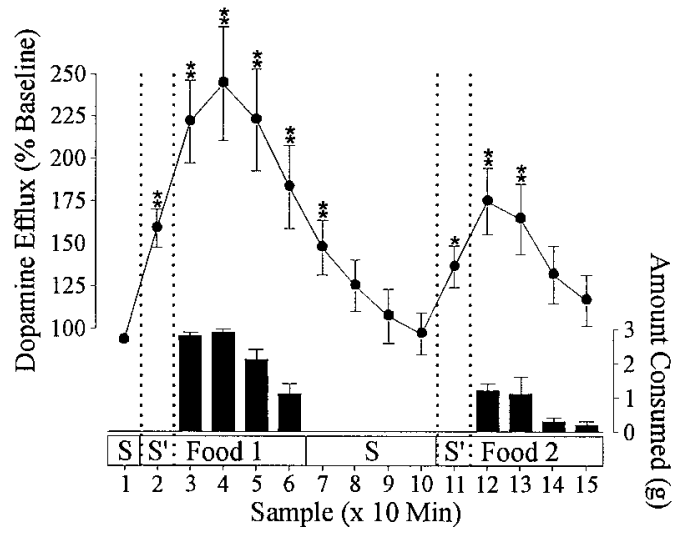

Figure 1. Changes in dopamine efflux in the medial prefrontal cortex (line graph) and amount of food consumed (bar graph) per 10 min during sensory-specific satiety experiments $(n=14)$. $A$, Same food condition, the effect of presenting food 1 during two consecutive meals. $B$, Different food condition, the effect of presenting food 1 during the first meal and food 2 during the second meal. Samples 1 and $7-10$ represent periods during which no food is present behind the screen $(S)$. Samples 2 and 11 represent the presence of food behind the screen $\left(S^{\prime}\right)$, and changes in dopamine efflux during these periods are highlighted by dashed lines. The last baseline value (sample 1) was used as the control mean in Dunnett's method of multiple comparisons $\left({ }^{* *} p<0.01 ;{ }^{*} p<0.05\right)$.

\section{RESULTS}

\section{Sensory-specific satiety effect}

Food-deprived rats were presented with two meals consisting of two different highly palatable foods (fruit-flavored cereal for the first meal and onion-flavored chips for the second meal or vice versa; different food condition) or the same food for both meals (same food condition). A sensory-specific satiety pattern of feeding was observed in all animals (Figs. $1 A, 2 A$, bar graphs). Animals quickly approached the food bin and began eating the first meal (food $1 ; 7.4 \pm 0.5 \mathrm{gm}$ in the same food condition, $7.9 \pm 0.4$ $\mathrm{gm}$ in the different food condition). The total amount of food consumed within any 40 min meal was not significantly affected by the type of food offered (e.g., during the first meal of the same food condition, animals consumed $7.6 \pm 0.8 \mathrm{gm}$ of fruit-flavored cereal and $7.1 \pm 0.7 \mathrm{gm}$ of onion-flavored chips). The gradual decrease in rate of consumption during the first meal indicated that animals were reaching satiety. If food 1 was offered again during a second meal (same food condition), animals took much longer to approach the food and occasionally sampled and consumed a significantly smaller amount than during the first meal $(1.0 \pm 0.3 \mathrm{gm} ; p<0.01)$. However, if animals were offered a novel palatable food (food 2, different food condition), they approached the food promptly and ate significantly more $(2.6 \pm 0.3 \mathrm{gm} ; p<$ 0.01 ) than when presented with food 1 a second time. A gradual decrease in amount consumed per $10 \mathrm{~min}$ period was also observed during the second meal (Figs. $1 B, 2 B$, bar graphs).

Before being tested on the sensory-specific satiety protocol with microdialysis, half of the rats had an opportunity to experience the same food condition, and the other half of the rats had the opportunity to experience the different food condition. In the microdialysis experiments, the type of prevous experience did not have a significant effect on either the amount of food consumed during the first $10 \mathrm{~min}$ of each meal $\left(F_{(1,26)}=0.494 ; p<0.49\right)$ or on the total amount of food consumed during each meal $\left(F_{(1,26)}\right.$ $=0.098 ; p<0.76)$. Similarly, the type of prior experience did not have a significant effect on changes in DA efflux in either the $\operatorname{mPFC}\left(F_{(1,12)}=0.155 ; p<0.70\right)$ or the $\operatorname{NAC}\left(F_{(1,12)}=0.654 ; p<\right.$ $0.43)$. In addition, there was no evidence of hemispheric differences in DA efflux in the $\operatorname{mPFC}\left(F_{(1,26)}=1.163 ; p<0.29\right)$ or in the NAC $\left(F_{(1,26)}=0.002 ; p<0.96\right)$. Specifically, changes in DA efflux in the left and right hemispheres of both brain regions were comparable during the same and different food conditions.

\section{Basal concentration of DA in MPFC and NAC dialysates}

The average concentration of DA (uncorrected for probe recovery) in the last three samples before presentation of food behind the screen was $0.18 \pm 0.01 \mathrm{~nm}$ in the $\mathrm{mPFC}$ and $3.16 \pm 0.19 \mathrm{~nm}$ in the NAC. These values likely reflect true basal concentrations of DA. First, animals were allowed an extended period (14-16 hr in the testing chamber after implantation of microdialysis probes) to habituate them to the testing chamber without the presence of food; second, samples were collected for at least $60 \mathrm{~min}$ before presentation of food behind the screen, during which there was a decreasing trend in DA levels, which eventually stabilized before the three samples used to estimate basal values.

\section{Changes in DA efflux in the mPFC}

The pattern of changes in DA efflux closely modeled the sensoryspecific satiety pattern of feeding. When the first meal consisting of food 1 was presented behind the screen, animals began scratching at or sniffing through the screen as well as locomoting around the testing chamber. These appetitive behaviors were accompanied by a significant rise in DA efflux $(+60 \%$ above baseline in the same food condition and $+63 \%$ in the different food condition; $p<0.01$; Fig. $1 A, B)$. When the screen was removed and animals began to eat, there was a further enhancement in DA efflux in the mPFC, reaching values greater than double the basal level $(+145 \%$ in the same food condition and $+154 \%$ in the different food condition; $p<0.01$ ) within the first $20 \mathrm{~min}$. Subsequently, as animals developed satiety for food 1, a gradual decline in DA levels mirrored the decreasing rate of food consumption. Reinsertion of the screen into the chamber and removal of any remaining food marked the start of a 40 min recess. During this time animals settled down in a corner of the chamber and engaged in periodic bouts of grooming while DA efflux returned to baseline levels.

Presentation of food 1 at the start of the second meal (same food condition) did not lead to significant levels of anticipatory 
Nucleus Accumbens

\section{A. Same Food Condition}

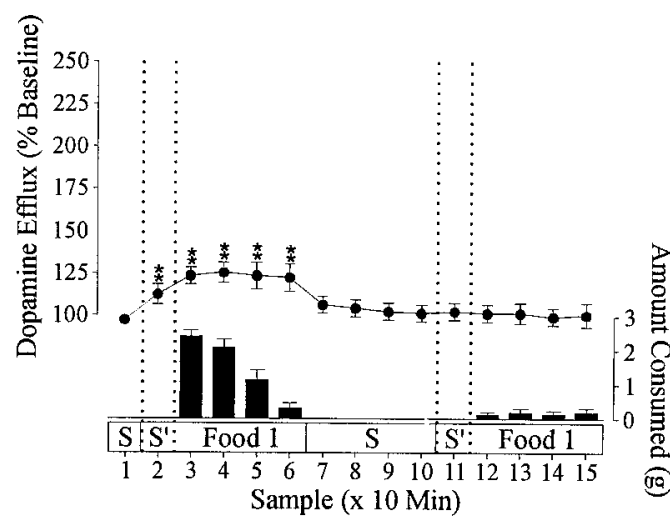

B. Different Food Condition

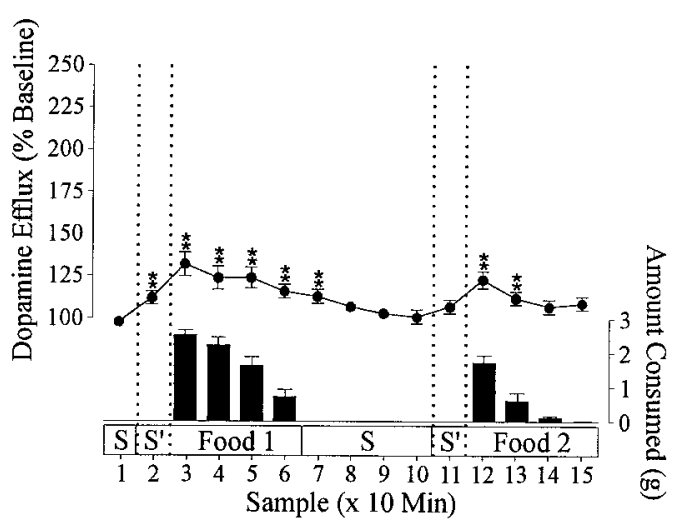

Figure 2. Changes in dopamine efflux in the nucleus accumbens (line graph) and amount of food consumed (bar graph) per $10 \mathrm{~min}$ during sensory-specific satiety experiments $(n=14)$. See Figure 1 legend for explanation of $A$ and $B$.

activity; animals remained inactive as they were during the recess, and DA efflux remained at baseline values (Fig. $1 A$ ). Removal of the screen was followed by a delayed approach response and occasional sampling of the food but no significant change in DA efflux. In marked contrast, presentation of food 2 behind the screen during the second meal (different food condition) led to increased appetitive behavioral responses and a significant increase in DA efflux in the mPFC $(+39 \%$; $p<0.05$; Fig. $1 B)$. When animals ate the novel food, DA levels continued to climb to $+80 \%(p<0.01)$ before descending to baseline levels again as animals reached satiety for food 2 .

\section{Changes in DA efflux in the NAC}

The pattern of DA efflux in the NAC closely mirrored the pattern observed in the mPFC, with two key exceptions. Similar to the DA response observed in the mPFC, presentation of food 1 during the first meal resulted in a significant increase in DA efflux during the appetitive phase $(+13 \%$ in the same food condition and $+12 \%$ in the different food condition; $p<0.05$; Fig. $2 A, B)$, which continued to rise during the consummatory phase $(+26 \%$ in the same food condition and $+32 \%$ in the different food condition within the first $20 \mathrm{~min} ; p<0.01)$. As in the mPFC, presentation of food 1 during the second meal was not accompa-
A

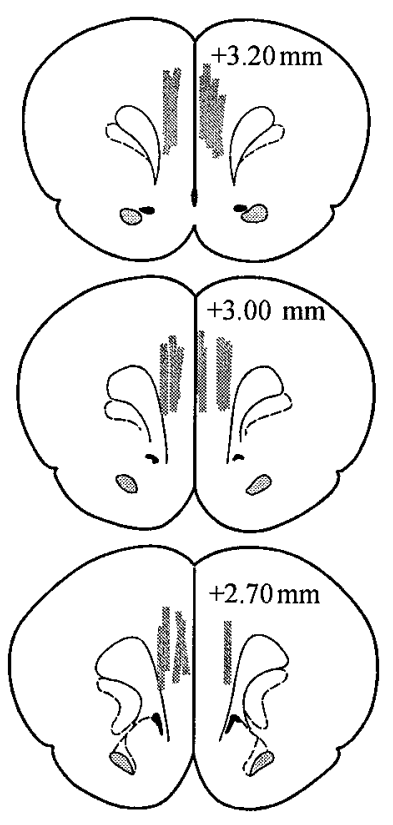

B

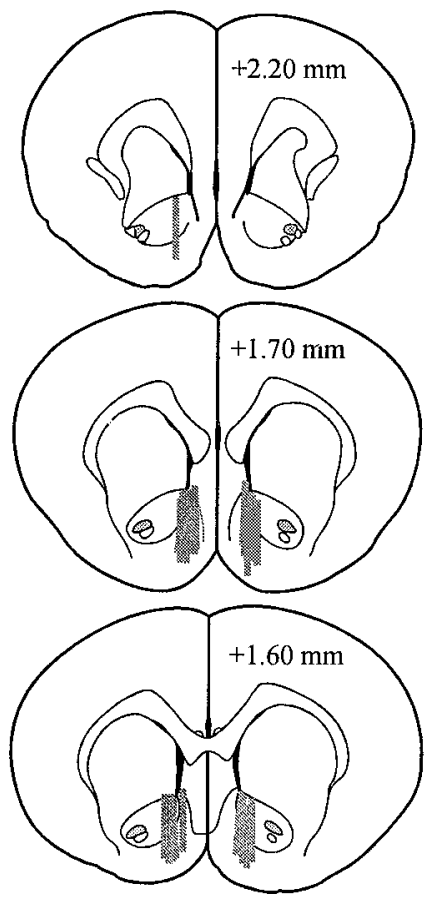

Figure 3. Location of microdialysis probes in the medial prefrontal cortex $(A)$ and nucleus accumbens $(B)$. Vertical lines represent the $2 \mathrm{~mm}$ dialyzing lengths of the probes. Drawings of coronal sections were adapted from those of Paxinos and Watson (1997). The distance from bregma is indicated for each section.

nied by any changes in NAC DA efflux (Fig. $2 A$ ). However, in contrast to the large DA efflux observed in the mPFC during the appetitive period of the second meal, presentation of a different palatable food behind the screen did not elicit a similar robust anticipatory DA response in the NAC (Fig. $2 B$ ). DA efflux in the NAC showed an insignificant increase during the appetitive phase $(+7 \%)$ and a small but significant increase during the consummatory phase $(+23 \%$; $p<0.01)$. It was noted that although the baseline concentration of DA in the NAC was $\sim 18$ times higher in the NAC than in the mPFC (see above), the magnitude of increase in DA efflux in the NAC, in comparison with that observed in the mPFC, was $\sim 5$ times smaller.

\section{Histology}

The tracts left by microdialysis probes were located in the prelimbic-infralimbic region of the $\mathrm{mPFC}(+3.2$ to $+2.7 \mathrm{~mm} \mathrm{AP}$ from bregma) or the shell-core region of the NAC $(+2.2$ to +1.6 mm AP from bregma; Fig. 3).

\section{DISCUSSION}

\section{Similarities in dopaminergic correlates of sensory- specific satiety in the MPFC and NAC}

The present findings confirm that when rats are food-deprived, DA efflux in both the mPFC and NAC is increased unconditionally in response to the presence of a novel food behind a screen and during ingestion of the initial meal. When rats display sensory-specific satiety, by sampling only small quantities of the original food presented as a second meal, there is no increase in DA efflux in either terminal region. In some respects this pattern of DA efflux in the NAC parallels the observations of Bassareo and Di Chiara (1997), when rats had eaten a novel palatable food 2-48 hr earlier. Another important finding is the second uncon- 
ditional increase in DA efflux in both the mPFC and NAC, when rats that are no longer food-deprived consume a different palatable meal. Together, these results point to major similarities in increases in DA efflux in the NAC and mPFC during sensoryspecific satiety.

The sensory-specific satiety pattern of DA activity is not limited to food-related stimuli. It was recently observed that DA efflux in the NAC is increased initially in male rats during copulation with a receptive female, is sustained for $\geq 60 \mathrm{~min}$, and declines during satiety. DA efflux is then increased unconditionally during renewed copulation with a second and novel female (Fiorino et al., 1997). The findings of studies for both sensoryspecific satiety for food and for a receptive mate are consistent with the incentive salience hypothesis (Berridge and Robinson, 1998), which predicts that changes in incentive value of stimuli should be accompanied by corresponding changes in mesotelencephalic DA activity. In the protocol used in the present study, the relative incentive values of the two palatable foods is changed by allowing hungry rats to feed to satiety on one of the foods. The results of a recent study suggest that as a food is consumed to satiety, its incentive value is gradually devalued through consummatory contact (Balleine and Dickinson, 1998). In our protocol, a similar devaluation process may have influenced DA efflux in the $\mathrm{mPFC}$ and NAC, because a gradual decrease in food intake was paralleled by a reduction in the magnitude of DA efflux during both meals in both brain regions. We suggest that as a consequence of this food-specific devaluation, presentation of the same food as a second meal was not accompanied by a change in DA efflux, and only a small quantity of the food was consumed. Furthermore, the food-specific devaluation is likely to have persisted into the second meal, producing a relative enhancement of the incentive properties of a novel food in comparison with the same food through "positive incentive contrast." This is an effect that arises when a stimulus of greater incentive value follows one of lesser value (Kentridge and Aggleton, 1993; Balleine and Dickinson, 1998). Thus, the relatively enhanced incentive value of the novel food may account for the rise in DA efflux and robust behavioral response during the second meal. If correct, this account implies that devaluation of a familiar food requires an "opportunity for incentive learning" (Balleine and Dickinson, 1998), whereas attribution of incentive salience to a food entirely or relatively novel does not require previous experience. In this regard, it is unlikely that the increases in DA efflux during exposure to novel odors or tastes are a result of previous training, because they were observed in all animals, including those that had never experienced two consecutive meals with different types of food.

\section{Differences in dopaminergic correlates of sensory- specific satiety in the MPFC and NAC}

The deprivation state of an animal is an important determinant of whether DA efflux in the NAC is elevated during feeding behavior, particularly during the appetitive phase (Wilson et al., 1995). In food-deprived animals, presentation of food in a perforated box or behind a screen (Wilson et al., 1995; Bassareo and Di Chiara, 1997) or even a cue associated with food (Phillips et al., 1993) increases DA efflux in the NAC. In nondeprived rats, however, no changes in DA efflux in the NAC are observed before the consumption of a palatable food or fluid (Wilson et al., 1995; Bassareo and Di Chiara, 1997). These observations suggest that, in the NAC, the magnitude of DA efflux during both the appetitive and consummatory phases is closely tied to the interaction between the deprivation or drive state of the organism and the conditioned or unconditioned incentive salience of biologically relevant rewards. This magnitude of DA activity may determine the general level of motivation and effort expended by an animal in seeking a reward (Salamone et al., 1997).

Recently, Bassareo and Di Chiara (1999) observed in nondeprived rats that DA efflux in the core, in comparison with the shell, region of the NAC is preferentially increased during exposure to a food-filled box, suggesting a possible difference in the role that DA may serve in the two regions. However, in the present study, these intra-accumbens differences in DA function are difficult to evaluate because most of the microdialysis probes were located across the shell-core boundaries (Fig. 3). Nevertheless, the fact that DA efflux in the NAC increased in the appetitive phase before the first meal indicates that our probe placements were located in a region of the NAC that responds to appetitive stimuli. In light of the findings of Bassareo and Di Chiara (1999), this increase in DA efflux may be attributed to an increase in the core portion of the NAC.

In contrast to the findings in the NAC, Bassareo and Di Chiara (1997) observed a significant elevation of DA efflux in the mPFC of nondeprived rats presented with a combination of olfactory and visual incentive stimuli. In the present study, a similar subtle but critical difference between the two brain regions is highlighted by the selective increase in DA efflux in the mPFC, but not in the NAC, of satiated rats when a different palatable food is presented behind a screen as the second meal. The results of both studies suggest that DA efflux in the mPFC is influenced solely by the incentive salience of rewards or by environmental stimuli associated with them (i.e., independently of the deprivation state of the animal). This feature of DA activity may be linked to the finding that neurons in the monkey dorsolateral prefrontal cortex show differential activity in response to different types of food, suggesting that these neurons can discriminate between preferred and less preferred foods (Watanabe, 1996). Therefore, it is possible that DA input to the mPFC contributes to the assignment of incentive value to behaviorally important stimuli in the environment, thereby mediating initial approach behaviors leading to consumption of a second meal.

\section{Mechanisms of sensory-specific satiety in the rat}

Studies of sensory-specific satiety by Rolls (1999) have shown in monkeys and humans that the sensory (olfactory, taste, and visual), as opposed to metabolic, properties of food are an important factor in determining food intake. This group also has concluded that neuronal activity, first in the primate orbitofrontal cortex and subsequently in the lateral hypothalamus, closely mirrors the rejection of sensory properties of a food eaten to satiety as well as the acceptance of the novel sensory properties and consumption of a different food (Rolls et al., 1986). Rolls (1999) has proposed that efferent projections from these brain regions to the ventral striatum may convey information about specific taste stimuli devalued by satiety to behavioral output systems. Although the neural circuitry involved in "computing" sensory-specific satiety in rats undoubtedly differs from primates (Rolls, 1999), the convergence of external sensory information and internal drive states in the rat brain must be a critical determinant of sensory-specific satiety.

In the rat, the amygdala is an important subcortical terminal region of the central taste pathways (Norgren, 1984) and the basolateral amygdala (BLA) appears to play an important role in assigning positive reward value to the sensory properties of food 
(Robbins and Everitt, 1996). It is also possible that these computations could be performed in the taste cortex of the rat, adjacent to the perirhinal regions of the prefrontal cortex (Pfaffman, 1982). There are several ways in which the assessment of the present incentive value of food stimuli may in turn modulate the efflux of $\mathrm{DA}$ in the mPFC and NAC. There is a direct projection from the BLA to the ventral tegmental area (Phillipson, 1979); however, there is no evidence that DA cell bodies that receive these afferents project, in turn, to the NAC. Alternatively, there is a direct projection from the BLA to the NAC that terminates in close apposition to tyrosine hydroxylase-containing varicosities (Johnson et al., 1994). This ultrastructural relationship may provide a potential site at which glutamatergic input from the BLA can increase DA efflux in the NAC (Floresco et al., 1998). The mechanism by which activity in the BLA may modulate DA efflux in the $\mathrm{mPFC}$ cannot be specified at this time, but this may involve the projection to the ventral tegmental area.

In summary, the present findings are consistent with the hypothesis that DA terminals in both the mPFC and NAC of the rat play an important role in the attribution of incentive value to sensory properties of biologically relevant stimuli and facilitate appropriate behavioral responses.

\section{REFERENCES}

Balleine B, Dickinson A (1998) The role of incentive learning in instrumental outcome revaluation by sensory-specific satiety. Anim Learn Behav 26:46-59.

Bassareo V, Di Chiara G (1997) Differential influence of associative and nonassociative learning mechanisms on the responsiveness of prefrontal and accumbal dopamine transmission to food stimuli in rats fed $a d$ libitum. J Neurosci 17:851-861.

Bassareo V, Di Chiara G (1999) Differential responsiveness of dopamine transmission to food-stimuli in nucleus accumbens shell/core compartments. Neuroscience 89:637-641.

Beninger RJ, Miller R (1998) Dopamine D1-like receptors and rewardrelated incentive learning. Neurosci Biobehav Rev 22:335-345.

Berridge KC (1991) Modulation of taste affect by hunger, caloric satiety, and sensory-specific satiety in the rat. Appetite 16:103-120.

Berridge KC, Robinson TE (1998) What is the role of dopamine in reward: hedonic impact, reward learning, or incentive salience? Brain Res Rev 28:309-369.

Blackburn JR, Pfaus JG, Phillips AG (1992) Dopamine functions in appetitive and defensive behaviours. Prog Neurobiol 39:247-279.

Cenci MA, Kalen P, Mandel RJ, Björklund A (1992) Regional differences in the regulation of dopamine and noradrenaline release in medial frontal cortex, nucleus accumbens and caudate-putamen: a microdialysis study in the rat. Brain Res 581:217-228.

Di Chiara G (1998) A motivational learning hypothesis of the role of mesolimbic dopamine in compulsive drug use. J Psychopharmacol 12:54-67.

Feenstra MGP, Botterblom MHA (1996) Rapid sampling of extracellular dopamine in the rat prefrontal cortex during food consumption, handling and exposure to novelty. Brain Res 742:17-24.

Fibiger HC, Phillips AG (1986) Reward, motivation, cognition: psychobiology of mesotelencephalic dopamine systems. In: Handbook of physiology: the nervous system (Bloom FE, ed), pp 647-676. Bethesda, MD: American Physiological Society.

Fiorino DF, Coury A, Phillips AG (1997) Dynamic changes in nucleus accumbens dopamine efflux during the Coolidge effect in male rats. J Neurosci 17:4849-4855.
Floresco SB, Yang CR, Phillips AG, Blaha CD (1998) Basolateral amygdala stimulation evokes glutamate receptor-dependent dopamine efflux in the nucleus accumbens of the anaesthetized rat. Eur $\mathbf{J}$ Neurosci 10:1241-1251.

Fuster JM (1991) The prefrontal cortex and its relation to behavior. Prog Brain Res 87:201-211.

Goldman-Rakic PS (1995) Architecture of the prefrontal cortex and the central executive. Proc Natl Acad Sci USA 769:71-83.

Hetherington MM, Rolls BJ (1996) Sensory-specific satiety: theoretical frameworks and central characteristics. In: Why we eat what we eat: the psychology of eating (Capaldi ED, ed), pp 267-290. Washington, DC: American Psychological Association.

Johnson J, Vickers Z (1992) Factors influencing sensory-specific satiety. Appetite 19:15-31.

Johnson LR, Aylward RLM, Hussain Z, Totterdell S (1994) Input from the amygdala to the rat nucleus accumbens: its relationship with tyrosine hydroxylase immunoreactivity and identified neurons. Neuroscience 61:851-865.

Kentridge RW, Aggleton JP (1993) Changes in cis(z)-flupentixolinduced dopamine blockade produce contrast effects in rats. Q J Exp Psychol 46B:113-127.

Kiyatkin EA (1995) Functional significance of mesolimbic dopamine. Neurosci Biobehav Rev 19:573-598.

Mogenson GJ, Brudzynski SM, Wu M, Yang CR, Yim CY (1993) From motivation to action: a review of dopaminergic regulation of limbic $\rightarrow$ nucleus accumbens $\rightarrow$ ventral pallidum $\rightarrow$ pedunculopontine nucleus circuitries involved in limbic-motor integration. In: Limbic motor circuits and neuropsychiatry (Kalivas PW, Barnes CD, eds), pp 193-236. Boca Raton, FL: CRC.

Norgren R (1984) Central neural mechanisms of taste. In: Handbook of physiology: the nervous system III, Sensory processes I (Darien-Smith I, ed), pp 1087-1128. Washington, DC: American Physiological Society.

Paxinos G, Watson C (1997) The rat brain in stereotaxic coordinates. Toronto: Academic.

Pfaffman C (1982) Taste: a model of incentive motivation. In: The physiological mechanisms of motivation (Pfaff DW, ed), pp 61-97. New York: Springer.

Phillips AG, Atkinson LJ, Blackburn JR, Blaha CD (1993) Increased extracellular dopamine in the nucleus accumbens of the rat elicited by a conditioned stimulus for food: an electrochemical study. Can J Physiol Pharmacol 71:387-393.

Phillipson OT (1979) Afferent projections to the ventral tegmental area of Tsai and interfascicular nucleus: a horseradish perioxidase study in the rat. J Comp Neurol 187:117-144.

Robbins TW, Everitt BJ (1996) Neurobehavioural mechanisms of reward and motivation. Curr Opin Neurobiol 6:228-236.

Rolls ET (1999) The brain and emotion. New York: Oxford UP.

Rolls ET, Rolls BJ (1997) Olfactory sensory-specific satiety in humans. Physiol Behav 61:461-473.

Rolls ET, Murzi E, Yaxley S, Thorpe SJ, Simpson SJ (1986) Sensoryspecific satiety: food-specific reduction in responsiveness of ventral forebrain neurons after feeding in the monkey. Brain Res 368:79-86.

Salamone JD, Cousins MS, Snyder BJ (1997) Behavioral functions of nucleus accumbens dopamine: empirical and conceptual problems with the anhedonia hypothesis. Neurosci Biobehav Rev 21:341-359.

Taber MT, Fibiger HC (1997) Activation of the mesocortical dopamine system by feeding: lack of a selective response to stress. Neuroscience 77:295-298.

Watanabe M (1996) Reward expectancy in primate prefrontal neurons. Nature 382:629-632.

Wilson C, Nomikos GG, Collu M, Fibiger HC (1995) Dopaminergic correlates of motivated behavior: importance of drive. J Neurosci 15:5169-5178. 